\title{
TTR
}

Traduction, terminologie, rédaction

Sergey Tyulenev. Applying Luhmann to Translation Studies. London and New York, Routledge, " Routledge Advances in Translation Studies », 2012, 235 p.

\section{Hélène Buzelin}

Volume 26, numéro 2, 2e semestre 2013

URI : https://id.erudit.org/iderudit/1037142ar

DOI : https://doi.org/10.7202/1037142ar

Aller au sommaire du numéro

\section{Éditeur(s)}

Association canadienne de traductologie

ISSN

0835-8443 (imprimé)

1708-2188 (numérique)

Découvrir la revue

Citer ce compte rendu

Buzelin, H. (2013). Compte rendu de [Sergey Tyulenev. Applying Luhmann to Translation Studies. London and New York, Routledge, « Routledge Advances in Translation Studies », 2012, 235 p.] TTR, 26(2), 276-281.

https://doi.org/10.7202/1037142ar d'utilisation que vous pouvez consulter en ligne.

https://apropos.erudit.org/fr/usagers/politique-dutilisation/ 
It must be noted that when conference proceedings or papers developed from presentations at conferences are published a certain amount of overlap and repetition is unavoidable. To illustrate, reference to the frequent presence of translators and interpreters in literature and film is made by several of the volume contributors, including Andres, Baer, Ben-Ari, Kurz, and Resch. The same can be said for the reference to the increased interest in this phenomenon on the part of translation studies scholars, as we see in Kaindl's "Going fictional," and Baer's "Interpreting Daniel Stein: Or what happens when fictional translators get translated," and to Walter Benjamin's essay "The Task of the Translator," which is cited by Apostolou and Kaindl, for example. However, given the strengths of the collection, these are mere quibbles.

Overall, the book offers many excellent features, not the least of which are the scope and range of the contributions. To quote Spitzl: "The volume in your hands offers no string of beads; it is rather a swirling collection of a multitude of approaches, perspectives and insights, which can-when put in motion (lecture)—turn into a vivid kaleidoscope" (p. 27). The editors of Transfiction are to be commended for providing a vehicle for critical observations on the works of such canonical writers as Cervantes, Borges, Voltaire, Conan Doyle, and Kafka, as well as on the fiction produced by professional translators and interpreters who reflect on the problems, limitations, and possibilities of their craft. This fine anthology deals with a phenomenon in literature and film that has important implications for Translation Studies.

Corrado Federici Brock University

\section{Sergey Tyulenev. Applying Lubmann to Translation Studies. London and New York, Routledge, «Routledge Advances in Translation Studies ", 2012, 235 p.}

Depuis une vingtaine d'années, les études descriptives (DTS) n'ont cessé d'emprunter à la sociologie. Parallèlement à celles de Pierre Bourdieu, d'Anthony Giddens et de Bruno Latour (pour ne citer que quelques noms), les idées de Niklas Luhmann se sont taillé une place grâce aux travaux d'Andreas Poltermann, de Theo Hermans, de Hans Vermeer et, plus récemment, de Sergey Tyulenev. Lobjectif du présent ouvrage consiste à appliquer 
le modèle de Luhmann à la traduction de la façon la plus «exhaustive» et la plus «satisfaisante» qui soit (p.1), les tentatives précédentes ayant été, selon l'auteur, fragmentaires ou déficientes, car souvent basées sur une mauvaise compréhension de ce modèle. La démonstration ne vise pas uniquement les phénomènes de traduction interlinguistique, mais les trois types de traduction définis par Jakobson, ce qui rend l'entreprise d'autant originale, intéressante et audacieuse.

«Luhmann replaces subject-centered reason with systems rationality» (p. 5). En un mot, la théorie des systèmes sociaux (TSS) développée par Luhmann a pour particularité d'envisager le monde social comme un ensemble de systèmes autopoiétiques. Dès l'introduction, l'auteur nous met en garde. La TSS est complexe. Pour autant, cet ouvrage ne cherche pas à la vulgariser ni même à la présenter. Il comprend cependant un glossaire très bien fait des notions clés de cette théorie. Ceux qui ne la connaîtraient pas sont donc invités (et auront intérêt) à consulter au préalable d'autres références. L'essai se compose de deux parties qui s'articulent autour de la notion de système.

La première partie pose les jalons. On y explore comment la traduction peut être considérée comme un système social à part entière (c'est-à-dire un système de communication) au même titre que la médecine, le droit, la politique ou l'économie. Selon l'auteur, «there is no doubt, that the history of translation over time is an evolution from a lower degree of organization to a higher degree of organization. Moreover, this evolution is a development from a lower degree of self-organization to a higher degree of selforganization» (p. 36). Dans la foulée, la professionnalisation de la traduction est évoquée comme un signe (parmi d'autres) que la traduction peut être considérée comme un système ou un champ au sens bourdieusien. Après avoir rappelé les arguments opposés, Tyulenev conclut qu'aucun ne pose un obstacle dans la mesure où «translation can be considered as a systemic social phenomenon based on its intrinsic properties [...]»(p.38). Autrement dit, le système renvoyant à lui-même (autoréférentialité) doit être défini en ses propres termes: "The possibility to describe translation as a system means that it is a system. If it were not, it would be impossible to construct translation as a system» (p. 80).

Cette première partie s'attache donc à déterminer les particularités dudit système: sa nature, sa fonction, ses propriétés, 
son code, etc. Ainsi, l'existence d'une réflexion sur la traduction, ou le fait que la traduction soit non seulement une pratique (observation de premier degré), mais aussi un discours sur la pratique (observation de deuxième degré) fournit une preuve qu'elle est bien un système. Ce système serait, par nature, un système de médiation ayant pour fonction d'assurer l'interaction entre chaque système et son environnement ainsi qu'entre les systèmes. Il s'agirait donc d'un "phénomène frontière ». ${ }^{1}$ Cette fonction est ce qui différentierait la traduction de tous les autres systèmes sociaux. Par ailleurs, comme tout système social, celui de la traduction se composerait d'événements de communication, mais ceux-ci auraient pour spécificité de faire intervenir non pas deux, mais trois parties. Autre propriété importante: la traduction requerrait deux côtés de valeur égale. Elle rechercherait et atteindrait son équilibre dans la tension entre ces deux côtés (cf. traduction littérale vs traduction libre). Le code, qui est toujours binaire, résiderait dans la distinction entre les événements de communication qui ont déjà fait et ceux qui n'ont pas encore fait l'objet d'une traduction/ médiation. Les seconds représenteraient la valeur marquée (cf. positive) du code, celle qui nourrit le système et lui permet de se reproduire. La partie se conclut sur une analogie surprenante entre le système de la médecine et celui de la traduction. ${ }^{2}$ Cette première partie crée, à la lecture, une curieuse impression d'étrangeté (dans les modes de raisonnement et concepts) et de familiarité (dans les idées avancées sur la traduction). Porté à «ne reconnaître que ce qu'il a appris au préalable à connaître» (Folkart, 1991, p. 310), le lecteur se demande alors si derrière l'apparence de nouveauté, le modèle proposé ne le reporterait pas quelques décennies en arrière (en renouant avec des postulats traductologiques aujourd'hui largement contestés) ou, au contraire, si ce modèle reprend les mêmes idées en leur donnant une autre portée. ${ }^{3}$

1. Les notions de système, de frontière et d'environnement renvoient à des entités purement conceptuelles et non physiques.

2. Selon l'auteur, les deux systèmes ont en commun de présenter un code dont la valeur positive correspond à une valeur négative dans le monde social: dans le premier cas il s'agit de la maladie et dans le second d'une absence d'information.

3. Si, comme le mentionne Tyulenev, Luhmann est parvenu à «dépasser» le fonctionnalisme, sa théorie en conserve néanmoins quelques traces, d'où cette impression de déjà vu. Selon Estelle Ferrarese, qui a contribué à faire connaître et à vulgariser la TSS en France, les nombreuses incompréhensions 
Une fois les bases énoncées, la deuxième partie approfondit la réflexion en analysant les particularités de la traduction comme sous-système social. Certains passages atteignent des niveaux d'abstraction vertigineux tandis que d'autres tentent d'interpréter quelques études de cas concrètes en termes «luhmanniens». Lauteur y montre aussi comment la traduction peut agir au sein du système politique, comment elle peut être un vecteur de conflit, le rôle qu'elle exerce dans l'évolution des systèmes, etc. La conclusion présente enfin une synthèse du travail, une recension des "pour et des contre» de la TSS suivie d'un plaidoyer dans lequel, après avoir spécifié que cette théorie nétait pas la seule façon d'étudier la traduction, l'auteur invite les traductologues à ouvrir leurs horizons, à envisager la contribution potentielle d'autres penseurs des sciences sociales et à étudier les liens entre des théories et concepts trop souvent perçus comme incompatibles. Cette dernière section est particulièrement riche, offrant de nombreuses avenues de recherche et de réflexion.

Si la première application traductologique de la théorie des systèmes sociaux de Luhmann remonte à une vingtaine d'années, celle-ci est, de loin, la plus fine, la plus approfondie, la plus exhaustive et certainement la plus ambitieuse produite jusqu'ici. $\mathrm{Ne}$ serait-ce que pour cette raison, le livre de Sergey Tyulenev intéressera tous ceux qui aspirent au développement d'une réflexion sociologique sur la traduction. Au chapitre des bémols, s'agissant d'un livre publié dans une collection de traductologie, on peut regretter que l'auteur n'ait pas pris le temps de présenter, de façon plus étayée et objective, l'auteur de la TSS: ses influences, sa trajectoire et sa position dans le champ de la sociologie. ${ }^{4}$ Cet aspect nest qu'effleuré dans l'introduction, où Luhmann apparait comme

que celle-ci peut susciter résultent en grande partie de l'«entêtement [de Luhmann] à recourir au vocabulaire du fonctionnalisme ou de la cybernétique, dont il a pourtant abandonné la plupart des paradigmes» (Ferrerase, 2012, italiques ajoutés).

4. Cela dit, comme la TSS fait peu de cas des actions individuelles et que Tyulenev considère les approches (socio)-biographiques d'un intérêt très limité, cette absence de contextualisation - ou plus exactement de réflexion sur les liens entre la trajectoire/position de Luhmann dans le champ des sciences sociales et les éléments clés de sa théorie- est cohérente et sans aucun doute délibérée. Elle nous rappelle qu'au-delà des points de recoupement possibles, la notion (luhmannienne) de système et celle (bourdieusienne) de champ sont malgré tout assez différentes. 
celui «who bravely exploded sociological boundaries» (p. 3), comme le premier à être parvenu à dépasser le fonctionnalisme de Talcott Parson (dont il se réclame néanmoins) en appliquant, à létude du monde social, des concepts empruntés à la biologie et la cybernétique (p.6). Par ailleurs, Luhmann s'est-il jamais intéressé aux phénomènes de traduction (restreinte ou élargie)? Si oui, qu'en a-t-il dit? Sinon, que penser de ce silence, sachant que la traduction/médiation serait finalement, selon Tyulenev, essentielle à l'existence même des systèmes? Son œuvre a-telle été traduite? Si oui, par qui, quand et dans quelles langues? Quelle en a été la réception dans ces langues? Ces questions simples et susceptibles d'intéresser les traductologues ne sont malheureusement pas explorées (sans doute parce quelles nétaient pas non plus essentielles à la démonstration). Un autre bémol est le caractère souvent circulaire des raisonnements. Cette circularité a beau être assumée et faire partie intégrante du système de pensée luhmannien, elle n'en reste pas moins dérangeante. En contrepartie, l'auteur établit de nombreux parallèles et liens entre la TSS et d'autres théories sociologiques. Ainsi les notions/idées des uns et des autres (Bourdieu, Latour, Even-Zohar, Habermas et bien d'autres) sont convoquées de façon aussi fréquente que ponctuelle lorsqu'elles peuvent servir la démonstration. Ces liens sont intéressants. Ils enrichissent le propos, mais, compte tenu des autres bémols mentionnés plus haut, ils semblent revêtir une fonction plus instrumentale que proprement «dialogique». Enfin, il est clair que la TSS permet d'envisager les phénomènes de traduction à un niveau d'abstraction jusque-là inégalé. Là réside son principal intérêt et la fascination quelle peut exercer. Cela dit, comme le rappelle le sociologue Johan Heilbron à propos de la traduction dans le «système mondial des livres»: «Il n'y a évidemment pas de transition simple et immédiate entre l'analyse d'un système mondial, celle d'une industrie éditoriale nationale, et celle de stratégies de traduction particulières» (Heilbron, 1999, p. 440, notre traduction). Si le système évoqué ici n'est pas de type luhmannien, cette réserve relative au passage d'un niveau d'analyse à l'autre s'applique néanmoins tout autant, voire plus encore, à la TSS qui se déploie et aspire à un niveau d'abstraction infiniment plus élevé. Autrement dit, à quel point la TSS permet-elle d'analyser et d'interpréter de façon satisfaisante des pratiques de traduction (restreinte ou élargie) complexes, et plus 
ou moins locales/globales? Quelles sont les limites et difficultés du passage d'un niveau (ou d'un ordre) d'analyse à l'autre? On peut regretter que l'auteur n'aborde pas directement ces questions. Cela dit, en tentant, à plusieurs reprises, de «traduire» en termes «luhmanniens » des études de cas traductologiques, il offre à chacun la possibilité de se forger une idée. Ainsi chaque lecteur pourra juger, par lui-même, de ce travail de «traduction». Ceux et celles qui, toute bigoterie mise à part, préfèrent expliquer la traduction en mobilisant les notions de sujet ou d'agent (plutôt qu'en invoquant la rationalité d'un système) seront sans doute mitigé(e)s tandis que les traductologues ayant un goût prononcé pour l'abstraction pure et qui sont en quête d'une théorie unificatrice à prétention universelle seront sûrement séduits. Au final, tous apprécieront le travail conceptuel, la richesse des idées, l'érudition, la rigueur, l'ambition et les analogies audacieuses qui font de cet essai résultat d'une réflexion aussi profonde qu'originale - un livre exigeant et une contribution importante à la sociologie de la traduction.

\section{Références}

Ferrarese, Estelle (2012). «À propos de Niklas Luhmann, Systèmes sociaux. Esquisse d'une théorie générale». Raison publique, 14 septembre 2012. [http://www.raison-publique.fr/auteur1445. html.]

Folkart, Barbara (1991). Le conflit des énonciations: traduction et discours rapporté. Québec, Les Éditions Balzac, «l'Univers des discours».

Heilbron, Johan (1999). «Toward a Sociology of Translation: Book Translations as a Cultural World-System». European Journal of Social Theory, 2, 4, p. 429-444.

HÉLÈne BuzeLIN Université de Montréal

Michelle Woods. Censoring Translation: Censorship, Theatre, and the Politics of Translation. Londres et New York, Continuum, 2012, 175 p.

Censoring Translation: Censorship, Theatre, and the Politics of Translation est le deuxième ouvrage publié par Michelle Woods, professeure agrégée au campus New Paltz de la State University of New York. Elle y poursuit une réflexion amorcée dans son premier ouvrage, Translating Milan Kundera, autour de la littérature 